\title{
Partial angle closure
}

\author{
R. MAPSTONE \\ From St. Paul's Eye Hospital, Liverpool
}

SUMMARY During the course of a negative provocative test for closed-angle glaucoma using pilocarpine and phenylephrine $60 \%$ of eyes develop significant reductions in outflow facility at some stage during the test. It is shown that these reductions can be explained by postulating the presence of partial-angle closure since: (1) A random sample (6) of 53 eyes showing an abnormal response subsequently had a peripheral iridectomy. On reprovoking they then behaved as normal eyes with a uniform increase in outflow. (2) Fifty-eight eyes that had a peripheral iridectomy for closed-angle glaucoma (spontaneous or induced) responded to provocative testing as do normal eyes.

Serial tonographies performed during the course of a positive provocative test in eyes at risk of developing closed-angle glaucoma indicate that in some eyes a significant proportion of the angle can close without the development of a pressure increase (Mapstone, 1977). If the test is continued almost all of the angle may close and pressure increase by $20 \mathrm{mmHg}$.

A priori there is no logical reason why angle closure during a provocative test should be an allor-none phenomenon. It is more reasonable to suppose that grades of closure occur between the two extremes of no closure and complete closure. Consequently a positive provocative test (an increase in intraocular pressure of $8 \mathrm{mmHg}$ or more) depends upon the extent and duration of angle closure. Theoretically, therefore, partial-angle closure may occur and yet a provocative test still be negative.

The obvious place to look for partial-angle closure is in eyes at risk of developing closed-angle glaucoma that do not develop a positive provocative test. The purpose of this paper is to investigate such a group and provide answers to the following questions: (1) Where does partial-angle closure occur? (2) How can it be diagnosed? (3) What are its clinical implications?

\section{Material and methods}

Fifty-three eyes from 53 patients with acute primary closed-angle glaucoma in the contralateral eye had negative provocative tests with pilocarpine and

Address for reprints: Mr R. Mapstone, St. Paul's Eye Hospital, Old Hall Street, Liverpool L3 9PF phenylephrine-that is, a pressure increase of less than $8 \mathrm{mmHg}$. They were reprovoked as follows: At zero hours the intraocular pressure was recorded, facility of outflow measured, and pilocarpine drops $2 \%$ and phenylephrine drops $10 \%$ were instilled. Approximately hour later an additional drop of phenylephrine $10 \%$ was instilled and the pressure recorded; $1 \frac{1}{2}$ hours from the start of the test the intraocular pressure was recorded, tonography was repeated, and a further dose of pilocarpine and phenylephrine instilled. Finally, 1 hour later (that is, $2 \frac{1}{2}$ hours from the start of the test) pressure was recorded and tonography repeated. The test was then terminated by the instillation of thymoxamine drops $\frac{1}{2} \%$.

When the results were analysed it was apparent that they formed a group with a normal response and another that was abnormal. The latter group was randomised and 6 selected for a peripheral iridectomy. Four months later they were reprovoked as described above.

Finally, 58 eyes from 42 patients that had had a peripheral iridectomy for either spontaneous closedangle glaucoma or a positive provocative test were provoked as described above.

Gonioscopy was not routinely done, since it soon became apparent that gonioscopy findings did not always equate with changes in outflow facility (see below).

\section{Results}

PROVOCATIVE TESTS IN 53 CONTRALATERAL EYES

Fig. 1 records the results of provocative testing in the 53 eyes and shows that the first dose of pilocar- 

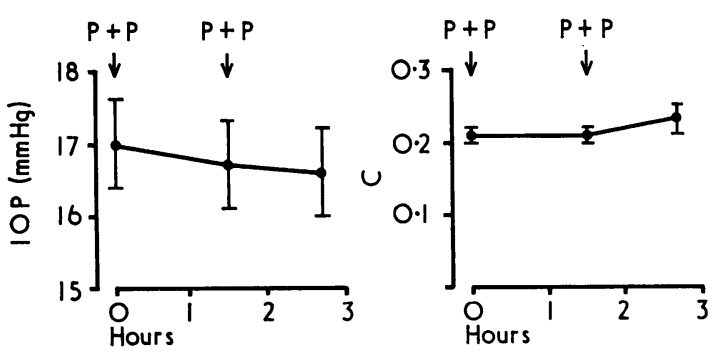

Fig. 1 Response of 53 eyes to provocative testing with pilocarpine and phenylephrine. Mean values and standard error recorded. $P+P=$ pilocarpine and phenylephrine

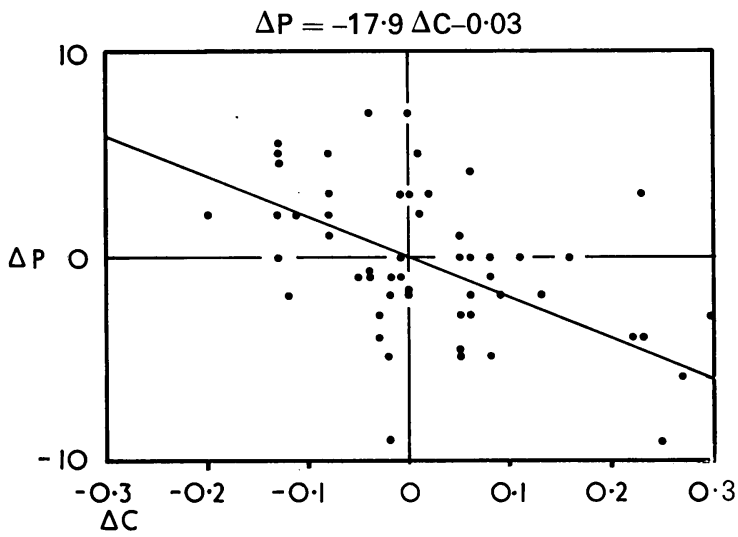

Fig. $2 A$ plot of the total change in pressure $(\Delta P)$ against the total change in $C(\Delta C)$ in the 53 eyes. The correlation coefficient was $-0 \cdot 51$

pine and phenylephrine produced a mean fall in pressure from 17 to $16.7 \mathrm{mmHg}$ and no change in outflow. The second dose of pilocarpine and phenylephrine produced a mean fall in pressure from 16.7 to $16.6 \mathrm{mmHg}$ and an increase in outflow (C) from 0.21 to 0.23 . Neither the overall change in pressure nor the total change in outflow was statistically significant.

Fig. 2 shows a plot of the total change in pressure for each eye against the total change in $C$. The equation of the regression line is $\Delta \mathrm{P}=-17.9 \Delta \mathrm{C}$ -0.03 . The correlation coefficient is -0.51 , which is significantly different from zero $(\mathrm{P}<0.001)$.

This analysis, however, hides the enormous variation in response. There are in fact 4 subgroups details of which are:

Group 1 (20 eyes). In this group the first dose of pilocarpine and phenylephrine produced a fall in pressure of $0.7 \mathrm{mmHg}$ and an increase in outflow of 0.07 (Fig. 3). The second dose of pilocarpine and phenylephrine produced an additional fall in pressure of $1.5 \mathrm{mmHg}$ and an increase in $\mathrm{C}$ of 0.06 . There

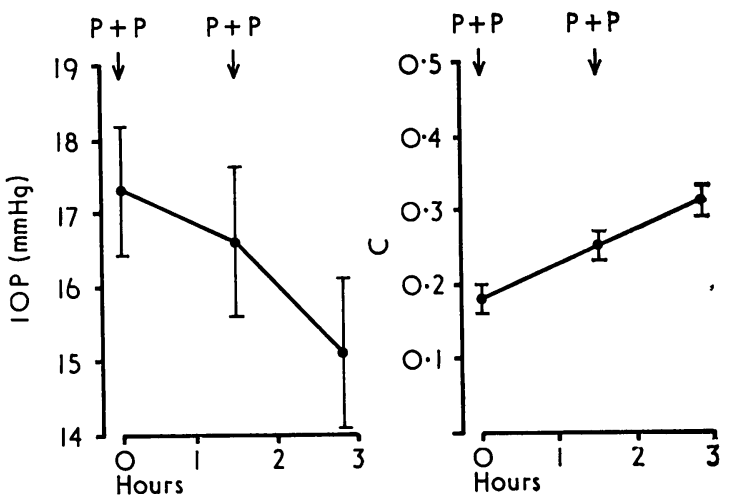

Fig. 3 Response of 20 eyes to provocative testing with pilocarpine and phenylephrine. Mean values and standard error recorded
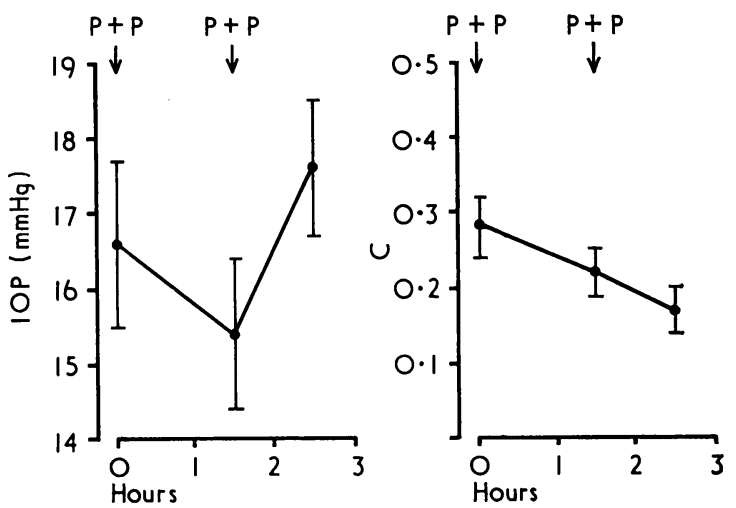

Fig. 4 Response of 10 eyes to provocative testing with pilocarpine and phenylephrine. Mcan values and standard error recorded

was therefore an overal decrease in pressure of $2.2 \mathrm{mmHg}$ and an overall increase in $\mathrm{C}$ of 0.13 .

Group 2 (10 eyes). Here the first dose of pilocarpine and phenylephrine produced a decrease in pressure of $1.2 \mathrm{mmHg}$ and a decrease in $\mathrm{C}$ of 0.06 (Fig. 4). The second dose of pilocarpine and phenylephrine produced an increase in pressure of $2.2 \mathrm{mmHg}$ and a further decrease in $\mathrm{C}$ of 0.05 . There was therefore an overall increase in pressure of $1 \mathrm{mmHg}$ and an overall decrease in $\mathrm{C}$ of $\mathbf{0} \cdot 11$.

Group 3 (10 eyes). The first dose of pilocarpine and phenylephrine produced a decrease in pressure of $1.1 \mathrm{mmHg}$ and an increase in $\mathrm{C}$ of 0.04 (Fig. 5). The second dose of pilocarpine and phenylephrine produced an increase in pressure of $1.6 \mathrm{mmHg}$ and a decrease in $\mathrm{C}$ of 0.05 . There was therefore an overall increase in pressure of $0.5 \mathrm{mmHg}$ and an overall decrease in C of 0.01 .

Group 4 (13 eyes). In the final group the first dose 


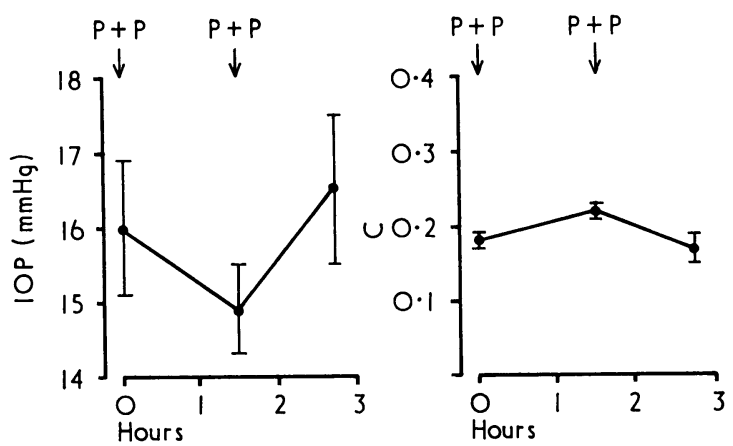

Fig. 5 Response of 10 eyes to provocative testing with pilocarpine and phenylephrine. Mean values and standard error recorded
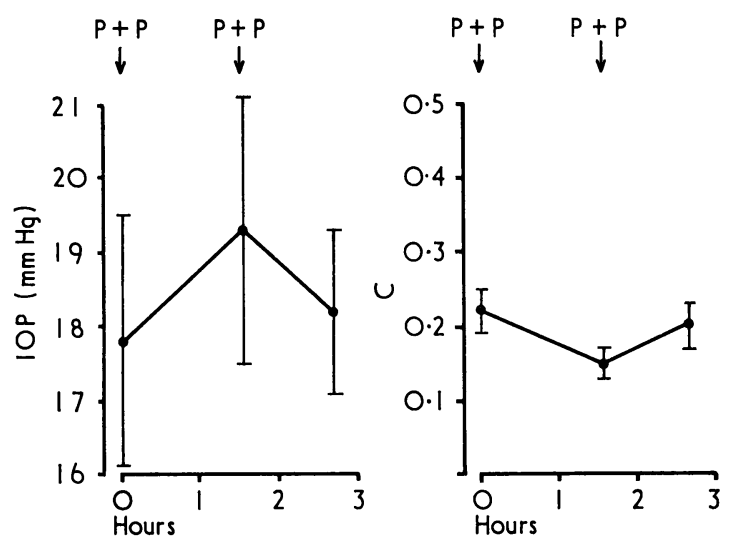

Fig. 6 Response of 13 eyes to provocative testing with pilocarpine and phenylephrine. Mean values and standard error recorded

of pilocarpine and phenylephrine produced an increase in pressure of $1.5 \mathrm{mmHg}$ and a decrease in $\mathrm{C}$ of 0.07 (Fig. 6). The second dose of pilocarpine and phenylephrine produced a decrease in pressure of $1.1 \mathrm{mmHg}$ and an increase in $\mathrm{C}$ of 0.06 . There was therefore an overall increase in pressure of $0.4 \mathrm{mmHg}$ and an overall decrease in $\mathrm{C}$ of 0.01 .

\section{EFFECT OF PERIPHERAL IRIDECTOMY IN SIX EYES}

The 6 randomly chosen eyes can be regarded as forming 2 groups, 1 before and 1 after peripheral iridectomy. There are therefore 2 analyses.

\section{Within group}

The result of a provocative test with pilocarpine and phenylephrine before peripheral iridectomy is shown in Fig. 7. Intraocular pressure increased on average $0.6 \mathrm{mmHg}$ (not significant), and $\mathrm{C}$ decreased by 0.08 (paired $t$ test, $0.01>\mathbf{P}>0.001$ ).
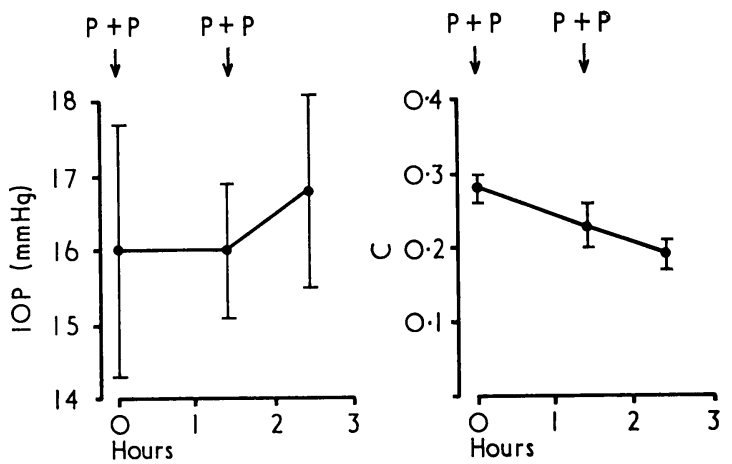

Fig. 7 Response of 6 eyes to provocative testing with pilocarpine and phenylephrine, before iridectomy. Mean values and standard error recorded

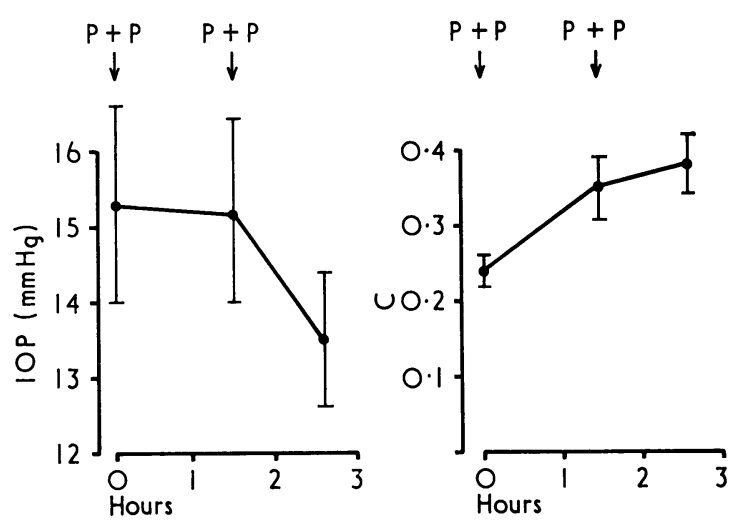

Fig. 8 Response of some 6 eyes as in Fig. 7 to provocative testing with pilocarpine and phenylephrine after iridectomy. Mean values and standard error recorded

The result of a provocative test in the same 6 eyes after a peripheral iridectomy is shown in Fig. 8 . Intraocular pressure decreased on average $1.8 \mathrm{mmHg}$ (not significant), and $\mathrm{C}$ increased by $0.13(0.01>\mathrm{P}$ $>0.001)$.

\section{Between group}

There was no significant difference in either the intraocular pressure or outflow facility of the 6 eyes at the start of the provocative test, before and after iridectomy. At the termination of the test, however, the mean pressure in the 6 eyes was significantly higher before iridectomy $(0.05>\mathrm{P}$ $>0.01)$, and $\mathrm{C}$ was significantly lower $(0.02>\mathrm{P}$ $>0 \cdot 01$ ).

PROVOCATIVE TESTS IN 58 Eyes AFTER A PERIPHERAL IRIDECTOMY

Fig. 9 records the results of provocative tests in 58 eyes that had had a peripheral iridectomy. The first 

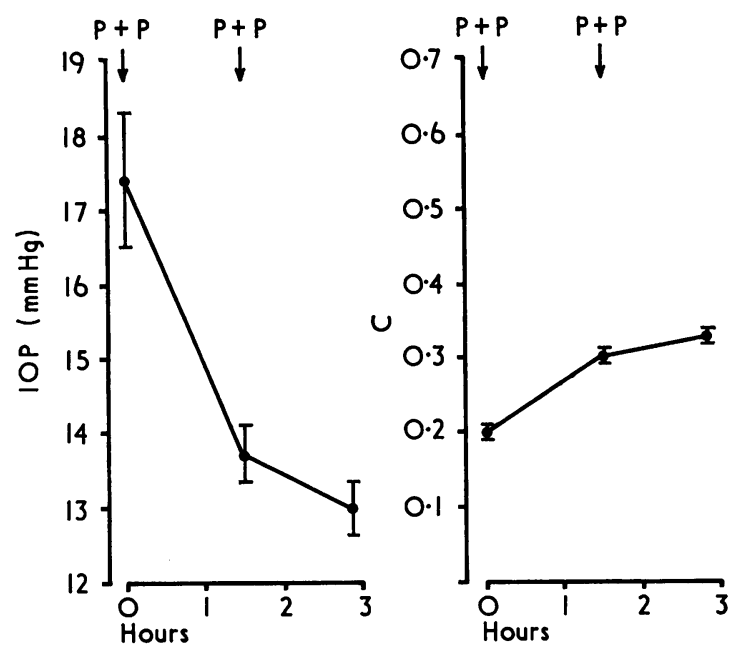

Fig. 9 Response of 58 eyes that had had a peripheral iridectomy to provocative testing with pilocarpine and phenylephrine. Mean values and standard error recorded

dose of pilocarpine and phenylephrine produced a pressure fall from 17.4 to $13.8 \mathrm{mmHg}$ and an increase in outflow from $0 \cdot 2$ to $0 \cdot 3$. The second dose of pilocarpine and phenylephrine again produced a pressure fall-from 13.7 to $13 \mathrm{mmHg}$, and an increase in $\mathrm{C}$-from 0.3 to 0.33 . Both the overall decrease in pressure and increase in $\mathrm{C}$ are statistically significant $(P<0.001)$.

If the behaviour of the 58 eyes in this group is compared with the 53 eyes at risk then the following results are obtained:

(a) There was no significant difference in either mean pressure or mean outflow at the start of the test-that is, before the instillation of the first dose of phenylephrine and pilocarpine $(\mathrm{z}=0.27$ and 0.55 , $P>0 \cdot 1$ ) (Figs. 1 and 9).

(b) At the time of instillation of the second dose of pilocarpine and phenylephrine pressure in the group that had not had a peripheral iridectomy was significantly higher than in the group that had $(\mathrm{z}=5.2, \mathrm{P}<0.001)$. On the other hand outflow facility was significantly lower $(z=4.4, P<0.001)$.

(c) At the termination of the test the same differences were present, for pressure $z=6.4$, $P<0.001$; for outflow $z=4.7, P<0.001$. That is, eyes in which a peripheral iridectomy had been done behaved as normal eyes do.

\section{Discussion}

A normal eye responds to provocative testing with pilocarpine and phenylephrine by developing a highly significant decrease in pressure and increase in outflow (Mapstone, 1977). This contrasts with the 53 contralateral eyes at risk described here in which neither the change in pressure nor the change in outflow was significant. In eyes at risk to closedangle glaucoma that develop a positive provocative test (pressure increase greater than $8 \mathrm{mmHg}$ ) 3 different outflow responses occurred (Mapstone, 1977): (1) One dose of pilocarpine and phenylephrine was sufficient to produce a positive result with a large reduction in outflow. (2) The first dose of pilocarpine and phenylephrine produced an increase in $\mathrm{C}$, whereas the second produced a decrease. (3) The first dose of pilocarpine and phenylephrine produced a decrease in $\mathrm{C}$, which was reduced still more by the second dose.

In the 53 eyes described here that had not had a peripheral iridectomy the outflow changes described in (2) and (3) occurred-without the development of a positive provocative test. In addition two other responses appeared: (4) A normal response in which both doses of pilocarpine and phenylephrine produced an increase in outflow. (5) An abnormal response in which the first dose of pilocarpine and phenylephrine produced a decrease in $\mathrm{C}$ and the second an increase.

Altogether, therefore, 4 different outflow responses were shown by the 53 eyes that had not had a peripheral iridectomy; $40 \%$ of these were as in normal eyes, but $60 \%$ were similar to the behaviour of eyes that developed a positive test. Yet in no instance was a positive test result-using the $8 \mathrm{mmHg}$ pressure rise yardstick-obtained. What then is the interpretation to be given to a reduction in outflow facility at some stage during the course of a negative provocative test with pilocarpine and phenylephrine?

Firstly, it could be argued that during the course of a provocative test homoeostatic drive or the drug combination used produced a variable reduction in outflow facility. This is unlikely because in normal eyes such behaviour was a rare event (Mapstone, 1977), and there is no reason to suppose that the outflow system in the 53 eyes described here is different from normal-apart from an increased probability of developing angle closure.

Secondly, the inaccuracies of tonography (Kronfeld, 1961; Fisher, 1970) could be advanced in explanation. However, normal eyes respond in a uniform way to this provocative test by developing an increase in $\mathrm{C}$. The observed difference is therefore a real one and-in the absence of angle closurecannot be explained by defects in methodology.

The third possibility is that partial-angle closure occurred at some stage during the test but that, because it was either spatially incomplete or temporally transient, a rise in pressure of $8 \mathrm{mmHg}$ 
did not occur. This explanation too is unlikely if the results of gonioscopy are taken into account. In some eyes there probably was partial-angle closure, but in others there was no doubt that immediately after tonography had shown a reduction in outflow facility gonioscopy revealed an open angle.

However, the demonstration of a gonioscopically open angle after tonography does not necessarily exclude the development of partial-angle closure during the provocative test. It could be argued that the system used for measuring outflow in some way distorts the anatomy of the anterior segment such that, in some eyes with narrow angles, the tonometer produces segmental irido-corneal contact and therefore low $\mathrm{C}$ readings, the implication being that the provocative test has made a narrow angle even narrower. As soon as the tonometer is removed the angle is open when viewed with a gonioscope. Again, it could be argued that partial-angle closure is a dynamic state of affairs with some of the angle closed only some of the time. If sufficient of the angle is closed for a sufficient proportion of time during tonography, the result will be an outflow deficit. Whether or not the tonometer is the proximate cause of irido-corneal contact is immaterial (it does not happen in normal eyes, nor in all eyes at risk); its mere presence indicates that part of the angle can close. It does not, therefore, necessarily follow that the measurement of a reduced outflow will be associated with an increased pressure. The length of time and amount of angle closed are the limiting factors. Neither is there reason to suppose that the angle will always appear partly closed at gonioscopy, since closure may be evanescent.

To argue these points is unnecessary, since the solution is readily found if it is accepted that a peripheral iridectomy in most instances prevents the development of angle closure. It is only necessary to do peripheral iridectomies in eyes that develop a reduction in outflow during a provocative test and then reprovoke after an interval. If the results of the provocative tests are now the same as in normal eyes, that would be some evidence in favour of accepting the reduction in outflow as indicating partial-angle closure at the time of tonography.

The result of provoking the 6 randomly chosen eyes before and after an iridectomy shows that after iridectomy the test results are the same as in normal eyes. A calculation that this is a chance phenomenon leads to a rejection of the null hypothesis, with a probability of less than 0.0000009 , that is, the hypothesis that the reductions in outflow are due to partial-angle closure is supported to a very highly significant degree. (The calculation assumes that eyes exhibiting an abnormal provocative test do not spontaneously convert to a normal one. To date there is no evidence that this happens in contralateral eyes at risk of developing closed-angle glaucoma to any significant degree.) It also necessarily follows that the reduced $\mathrm{C}$ values cannot be explained as due to homoeostatic drive or as a direct pilocarpine/ phenylephrine effect on trabecular meshwork; since if they singly, or in combination, produced this effect before iridectomy there is no reason to suppose that the same effect would not be produced after iridectomy. Finally, the response of the 58 eyes that had a peripheral iridectomy was in every way similar to that of normal eyes with a significant reduction in pressure and increase in outflow, the implication being that a peripheral iridectomy prevented the development of angle closure.

The evidence for accepting that the provocative tests described here demonstrate partial-angle closure seems, therefore, compelling and can be briefly summarised: Normal eyes respond to provocative testing with pilocarpine and phenylephrine by developing a decrease in pressure and an increase in C. Eyes at risk to closed-angle glaucoma develop either a positive provocative test (increase in pressure greater than $8 \mathrm{mmHg}$, reduction in $\mathrm{C}$ to almost complete angle closure), or a negative test (increase in pressure of less than $8 \mathrm{mmHg}$ ). Of the latter, $40 \%$ respond in a normal fashion (see above) and $60 \%$ develop reductions in outflow at some time during the test, but the increase in pressure is less than $8 \mathrm{mmHg}$.

If a peripheral iridectomy is done in a sample of these eyes, the provocative test result is as for normal eyes with a uniform increase in outflow. It is therefore improbable that the reductions in outflow can be explained on the basis of homoeostatic drive or as drug induced. In normal eyes there is no correlation between the increase in outflow and reduction in intraocular pressure (Mapstone, 1977). In the 53 eyes described here, there is a highly significant negative linear correlation between the change in pressure and the change in outflow, the most probable explanation being that partial-angle closure occurs.

Finally, eyes that have had a peripheral iridectomy for closed-angle glaucoma respond in the same way as eyes with no evidence of glaucoma. They are therefore significantly different from contralateral eyes that develop a 'negative' provocative test but reduced $\mathrm{C}$ values, and this is most probably explained by the absence of angle closure.

The first two questions mentioned in the introduction (where does partial-angle closure occur; and how can it be demonstrated?) have been partly answered. The third question (what are its clinical implications?), can only be speculative. The 53 patients described form part of a group that have 
been accumulated over the past 9 years. They have in common the fact that although at risk of developing closed-angle glaucoma (because the contralateral eye has had an acute attack) they nevertheless respond to provocative testing with an increase in pressure of less than $8 \mathrm{mmHg}$. Throughout this period they have received no treatment. Their prospective study may eventually provide evidence of relevance to the management of eyes with narrow angles, and this was the reason for not doing peripheral iridectomies. It has been the practice to provoke these eyes at roughly yearly intervals, and it is apparent that approximately $10 \%$ do, in fact, eventually develop positive provocative tests. The first implication of partial-angle closure is therefore that some such eyes may eventually develop positive provocative tests and presumably spontaneous closed-angle glaucoma too.

The second implication is that in $90 \%$ of contralateral eyes at risk an abnormality can be demonstrated by provocative testing with pilocarpine and phenylephrine-either an increase in pressure of greater than $8 \mathrm{mmHg}$ or a reduction in outflow facility at some stage during the test.

There is, however, a much wider and more important implication of partial-angle closure, for if some of the angle is closed some of the time then the clinical appearance may simply be ocular hypertension, the inference being that partial-angle closure may be the causal mechanism in some patients with ocular hypertension. These points are explored in subsequent papers.

I am grateful to colleagues who referred patients for study, to Mr M. C. C. Tweedie, Department of Biostatistics, University of Liverpool, for statistical advice, and to Mrs E. Tubb for secretarial help.

\section{References}

Fisher, R. F., Carpenter, R. G., and Wheeler, C. (1970). British Journal of Ophthalmology, 54, 217.

Kronfeld, P. C. (1961). Transactions of the American Academy of Ophthalmology and Otolaryngology, 65, 133.

Mapstone, R. (1977). British Journal of Ophthalmology, 61, 